\title{
Effectiveness of Chin-tuck Maneuver to Facilitate Swallowing in Neurologic Dysphagia
}

\author{
Mariana Saconato $^{1}$ Brasilia Maria Chiari ${ }^{1}$ Henrique Manoel Lederman ${ }^{2}$ Maria Inês Rebelo Gonçalves ${ }^{1}$ \\ ${ }^{1}$ Department of Phonoaudiology, Universidade Federal de São Paulo, \\ São Paulo, São Paulo, Brazil \\ 2 Image Diagnostics, Universidade Federal de São Paulo, São Paulo, \\ São Paulo, Brazil \\ Address for correspondence Mariana Saconato, MSc, Department of \\ Phonoaudiology, Universidade Federal de São Paulo, R: Pedro de \\ Toledo, 902 São Paulo 04023-900, Brazil \\ (e-mail: marisac35@yahoo.com.br).
}

Int Arch Otorhinolaryngol 2016;20:13-17.

\begin{abstract}
Introduction The chin-tuck maneuver is the most frequently employed postural maneuver in the treatment of neurogenic oropharyngeal dysphagia caused by encephalic vascular strokes and degenerative diseases.

Objective The purpose of this study was to investigate the effectiveness of this maneuver in patients with neurogenic dysphagia and factors that could interfere in it. Methods In this retrospective cohort, we analyzed the medical files and videofluoroscopy exams of 35 patients ( 19 male $-54 \%$ and 16 female $-46 \%$; age range between 20 and 89 years old; mean $=69$ years).

Results The results suggest that the effectiveness of chin-tuck maneuver is related to

Keywords

- deglutition

- fluoroscopy

- deglutition disorders

- dysphagia the overall degree of dysphagia: the more severe the dysphagia, the less effective the maneuver.

Conclusion Chin-tuck maneuver should benefit dysphagic patients with delay in the swallowing trigger, reduced laryngeal elevation, and difficulties to swallow liquids, but is not the best compensatory strategy for patients with severe dysphagia.
\end{abstract}

\section{Introduction}

The so-called chin-tuck maneuver (CTM) does not modify the physiology of deglutition; however, it does change pharyngeal dimensions as to direct the bolus toward the pharynx and esophagus. It is the most frequently employed postural maneuver in the treatment of neurogenic oropharyngeal dysphagia caused by encephalic vascular strokes and degenerative diseases. ${ }^{1-7}$

During the execution of this maneuver we observe narrowing of the laryngeal portion of the pharynx, which often compensates for delay of glottic closure during swallowing and favors a shift of the tongue base toward the posterior wall of the pharynx. ${ }^{1,5-8}$ The literature supports that patients who experience delays in the swallowing trigger usually benefit from this maneuver, as it significantly reduces the risk of food aspiration immediately prior to deglutition. ${ }^{2}$ Such positive effects are however controversial, since some authors have reported no significant modifications in what concerns pharyngeal contraction and even point out some disadvantages concerning the bolus flow from the oral cavity to the pharynx..$^{3-6,9,10}$

Although the CTM is widely used to prevent aspiration in patients with dysphagia, some studies question its effectiveness. An evidence-based systematic literature review on the impact of dysphagia treatment on populations with neurological disorders found that CTM was effective in 8 to $50 \%$ of cases. $^{7,8}$

In a study involving patients with Parkinson's disease and dementia, CTM was less effective when compared with the intake of thickened liquids. ${ }^{11}$

Considering the controversy on its effects and the relevance of this maneuver as one of the first choices for swallowing rehabilitation, ${ }^{8,12-14}$ we investigated factors that received

January 4, 2015

accepted

June 26, 2015

published online

October 20, 2015
DOI http://dx.doi.org/

$10.1055 / \mathrm{s}-0035-1564721$. ISSN 1809-9777.
Copyright (c) 2016 by Thieme Publicações License terms

Ltda, Rio de Janeiro, Brazil 
could interfere with the effectiveness of this maneuver in patients with neurogenic dysphagia.

\section{Methods}

In this retrospective cohort, we analyzed the medical files and videofluoroscopy exams of 35 patients (study group) diagnosed with neurogenic dysphagia that showed penetration and/or laryngotracheal aspiration and, for this reason, performed the CTM maneuver. The exams were performed between June 2008 and August 2010, in a major Brazilian university hospital. The Ethics in Research Committee of the institution of origin approved this study under number 1049/ 10. All patients were under treatment in the Clinic for Functional Rehabilitation of Swallowing Disorders of the University Hospital, and have signed a formal consent to participate in this study.

For the 35 selected patients ( 19 male - $54 \%$ and 16 female $46 \%$; age range between 20 and 89 years old, mean $=69$ ) we described the incidence of the etiologies for neurogenic dysphagia: Vascular Encephalic Stroke (69\%), Parkinson's disease (11\%), Multiple Sclerosis (11\%), and Myasthenia Gravis (9\%). We eliminated any incomplete and unintelligible files and poor quality videofluoroscopic images.

From the physical exams we considered the presence of abnormalities in oropharyngeal functions, which are defined by alterations in mobility, sensibility, tonus, muscular strength, movement amplitude of the lips, tongue, soft palate and mandible.

To perform the videofluoroscopic exams, patients are requested to remain seated and swallow barium-contrasted food in different consistencies: thin liquid, liquid thickened to consistencies of nectar, honey, and pudding ( $5 \mathrm{ml}$ and $10 \mathrm{ml}$ ), and solid. However, for this study, we considered only the liquid and thickened liquid consistencies, since they were the only ones that led to penetration/aspiration.

In the pharyngeal phase, we evaluated laryngeal elevation using the four finger method, by which the index finger is placed submentally, the middle finger is placed on the hyoid, and the last two fingers are placed on the superior and inferior borders of the thyroid cartilage. Poor laryngeal elevation may indicate reduced laryngeal elevation. ${ }^{11}$

The triggering or onset of pharyngeal swallowing is considered normal below $0.24 \mathrm{~s}$. It is delayed whenever laryngeal elevation and closure of the laryngeal aditus occur after the bolus transit through the region between the oral cavity and the oral portion of the pharynx..$^{8,11-13}$ The prevention of airway invasion during swallowing is laryngeal vestibule closure, which is achieved when the hyoid and larynx approximate. The approximation of these two structures results in the compression of the median thyrohyoid tissue, which bulges backward as a result, closing the laryngeal entrance. ${ }^{12}$

Laryngeal penetration and tracheal aspiration are the presence of contrasted food immediately above or below the vocal folds, respectively. ${ }^{1,8,11}$ To classify the severity of dysphagia, we used the O'Neil scale. ${ }^{15}$

\section{The Dysphagia Outcome and Severity Scale ${ }^{15}$ Full Per-oral (PO) Nutrition: Normal Diet}

Level 7: Normal in all Situations

Normal diet

No strategies or extra time needed

Level 6: Within Functional Limits/Modified Independence Normal diet, functional swallow

Patient may have mild oral or pharyngeal delay, retention, or trace epiglottal undercoating, but independently and spontaneously compensates/clears

May need extra time for meal

Has no aspiration or penetration across consistencies

Full PO: Modified diet and/or independence

Level 5: Mild Dysphagia: Distant Supervision, May Need One Diet Consistency Restricted

May exhibit one or more of the following:

Aspiration of thin liquids only but with strong reflexive cough to clear completely

Airway penetration midway to cords with one or more consistency or to cords with one consistency but clears spontaneously

Retention in pharynx that is cleared spontaneously

Mild oral dysphagia with reduced mastication and/or oral retention that is cleared spontaneously

Level 4: Mild-moderate Dysphagia: Intermittent Supervision/Cueing, One or Two Consistencies Restricted May exhibit one or more of the following:

Retention in pharynx cleared with cue

Retention in the oral cavity that is cleared with cue

Aspiration with one consistency, with weak or no reflexive cough

Or airway penetration to the level of the vocal cords with cough with two consistencies

Or airway penetration to the level of the vocal cords without cought with one consistency

Level 3: Moderate Dysphagia: Total Assist, Supervision, or Strategies, Two or More Diet Consistencies Restricted May exhibit one or more of the following:

Moderate retention in pharynx, cleared with cue

Moderate retention in oral cavity, cleared with cue

Airway penetration to the level of the vocal cords without cough with two or more consistencies

Or aspiration with two consistencies, with weak or no reflexive cough

Or aspiration with one consistency, no cough and airway penetration to cords with one, no cough

Non-oral nutrition necessary

Level 2: Moderately Severe Dysphagia: Maximum Assistance or Use of Strategies with Partial PO Only (Tolerates at Least One Consistency Safely with Total Use of Strategies)

May exhibit one or more of the following: 
Table 1 Videofluoroscopic abnormalities in the study group

\begin{tabular}{|l|l|l|}
\hline Videofluoroscopic findings (\%) & CDM $(\mathbf{n}=\mathbf{3 5})$ & $\%$ \\
\hline Delayed swallowing trigger & 27 & $77 \%$ \\
\hline Laryngeal elevation alteration & 27 & $77 \%$ \\
\hline Oropharyngeal functions alteration & 16 & $46 \%$ \\
\hline Liquid penetration & 21 & $60 \%$ \\
\hline Nectar penetration & 17 & $49 \%$ \\
\hline Pudding penetration & 14 & $40 \%$ \\
\hline Liquid aspiration & 12 & $34 \%$ \\
\hline Nectar aspiration & 7 & $20 \%$ \\
\hline Pudding aspiration & 8 & $23 \%$ \\
\hline
\end{tabular}

Abbreviation: CDP, chin-down maneuver.

Severe retention in pharynx, unable to clear or needs multiple cues

Severe oral stage bolus loss or retention, unable to clear or needs multiple cues

Aspiration with two or more consistencies, no reflexive cough, weak volitional cough

Or aspiration with one or more consistency, no cough and airway penetration to cords with one or more consistency, no cough

Level 1: Severe Dysphagia: NPO, Unable to Tolerate any P. O. Safely

May exhibit one or more of the following:

Severe retention in pharynx, unable to clear

Severe oral stage bolus loss or retention, unable to clear

Silent aspiration with two or more consistencies, nonfunctional volitional cough

Or unable to swallow

Correlations of such variables and observable modifications during the chin-tuck maneuver were statistically veri- fied by means of chi-square and logistic regression tests, with significance level of $5 \%$.

\section{Results}

- Table 1 shows the videofluoroscopic abnormalities in the study group. The following changes were observed: delayed swallowing trigger, laryngeal elevation alteration, oropharyngeal functions alteration, liquid penetration, nectar penetration, pudding penetration, liquid aspiration, nectar aspiration and pudding aspiration.

In this study, $77 \%$ of the patients presented delayed swallowing trigger and altered laryngeal elevation, while $46 \%$ showed oropharyngeal functions alteration. Regarding penetration of liquid, thickened liquid nectar, and pudding consistencies, we observed prevalence rates of $60 \%, 49 \%$, and $40 \%$, respectively. Liquid aspiration was present in $34 \%$ of patients, while nectar and pudding aspiration was found in $20 \%$ and $23 \%$ of patients, respectively.

-Table 2 shows videofluoroscopic abnormalities in the study group according to penetration/aspiration prevention or not prevention during chin-down maneuver.

We can observe from this table that, in patients showing delayed swallowing trigger, the CTM prevented laryngotracheal aspiration in $81 \%$, a statistically significant result $(p=0.002)$.

In the cases with laryngeal elevation alteration and oropharyngeal functions alteration the CTM was effective in $74 \%$ and $75 \%$ of the sample respectively, with no statistically significant results.

On patients presenting laryngeal penetration for liquid consistency, the CTM was effective in $76 \%$ of the sample, with statistically significant results $(p=0.009)$. On patients with laryngeal penetration for the nectar and pudding consistency, the CTM was effective in $71 \%$ and $64 \%$, respectively, with no statistically significant results.

Regarding tracheal aspiration for liquid, liquid nectar-thick and liquid pudding-thick, the CTM was effective in $83 \%, 57 \%$

Table 2 Videofluoroscopic abnormalities in the study group according to prevention or not prevention during chin-down maneuver

\begin{tabular}{|c|c|c|c|c|c|}
\hline Videofluoroscopic findings (\%) & $\begin{array}{l}\text { Aspiration } \\
\text { prevention } \\
\mathrm{N}=24\end{array}$ & $\begin{array}{l}\text { No aspiration } \\
\text { prevention } \\
\mathrm{N}=11\end{array}$ & Total Individuals & Prevention & p-value \\
\hline Delayed swallowing trigger & 22 & 5 & 27 & $81 \%$ & $0.002^{*}$ \\
\hline Laryngeal elevation alteration & 20 & 7 & 27 & $74 \%$ & 0.957 \\
\hline Oropharyngeal functions alteration & 12 & 4 & 16 & $75 \%$ & 0.279 \\
\hline Liquid penetration & 16 & 5 & 21 & $76 \%$ & $0.009^{*}$ \\
\hline Nectar penetration & 12 & 5 & 17 & $71 \%$ & 0.182 \\
\hline Pudding penetration & 9 & 5 & 14 & $64 \%$ & 0.116 \\
\hline Liquid aspiration & 10 & 2 & 12 & $83 \%$ & 0.903 \\
\hline Nectar aspiration & 4 & 3 & 7 & $57 \%$ & 0.187 \\
\hline Pudding aspiration & 3 & 5 & 8 & $38 \%$ & 1.187 \\
\hline
\end{tabular}


Table 3 Relationship between degree of dysphagia and chin-down maneuver effectiveness

\begin{tabular}{|l|l|l|l|l|l|}
\hline Degree of dysphagia & $\begin{array}{l}\text { Aspiration prevention } \\
\mathbf{N}=\mathbf{2 4}\end{array}$ & $\begin{array}{l}\text { No aspiration prevention } \\
\mathbf{N}=\mathbf{1 1}\end{array}$ & Total Individuals & Prevention & p-value \\
\hline Mild dysphagia & 3 & 0 & 3 & $100 \%$ & - \\
\hline Moderate dysphagia & 8 & 12 & 20 & $40 \%$ & $\mathbf{0 . 0 0 1}^{*}$ \\
\hline Severe dysphagia & 1 & 11 & 12 & $8 \%$ & - \\
\hline
\end{tabular}

*statistical significance ( $\mathrm{p}$-value $<0.05)$.

and $38 \%$ of the sample respectively, with no statistically significant results.

- Table 3 shows the relationship between degree of dysphagia and CTM effectiveness. Our findings indicate that the CTM is effective in moderate cases of dysphagia, with an effectiveness of $40 \%$ in patients with tracheal aspiration, a statistically significant result $(p=0.001)$.

\section{Discussion}

In this study we observed that the presence of alterations in oropharyngeal structures was a significant interference factor for the effectiveness of the CTM. Indeed, especially in cases of reduced strength of facial and masticatory muscles with weak or incomplete lip sealing during swallowing causing extraoral spills, this maneuver can be extremely difficult to perform. Some patients with reduced intra-oral sensibility and reduced tongue mobility may present poor oral motor control of the bolus, which also causes problems when performing the CTM. Some authors advocate that the anticipated flow of bolus toward the piriforms recesses may also interfere with the effectiveness of the CTM. ${ }^{8,12-14}$

The positioning of the head during this maneuver approximates the larynx to the tongue base and promotes upper airway protection, thus simulating normal laryngeal vertical excursion. This explains why the normality of this variable does not interfere with the effectiveness of this maneuver, and most patients with reduced laryngeal elevation indeed benefit from it.

The literature reports that possible interactions of variables, such as laryngeal vertical movement and oropharyngeal alterations causing dysphagia, ${ }^{12}$ weakened tongue movements, and delayed swallowing trigger may desynchronize oral and pharyngeal phases of swallowing. ${ }^{13}$

As for delayed swallowing trigger, it was a factor of interference in the effect of the maneuver $(p=0.002)$. Some authors recommend chin-tuck maneuver to patients presenting delayed swallowing trigger, incomplete laryngeal closure, and insufficient posterior displacement of the tongue base, ${ }^{1,2,6-10,12,14}$ since this head positioning favors food collection inside the valleculae and prevents anticipated spill into the pharynx. ${ }^{1,5-7,15}$

Many studies report the high incidence of alterations in the swallowing trigger in patients with neurogenic dysphagia. ${ }^{16-19}$ The lower excitability threshold is noticeable whenever swallowing is not triggered in presence of stimulus. ${ }^{5,7,8,10,19}$ Patients with delayed swallowing trigger may present incoordination or absence of laryngeal elevation. ${ }^{14-17,20}$

In what concerns laryngeal penetration and tracheal aspiration, we observed that the CTM is especially effective in patients with difficulties to swallow thin liquid. Similar results are reported in the literature, showing that CTM does increase swallowing safety in patients with dysphagia for liquids, ${ }^{7}$ as it widens the vallecular space, narrows the laryngeal aditus, and approximates the arytenoid cartilages to the epiglottis. ${ }^{1,2,8,10,12,19}$

It is well known in the literature that neurogenic dysphagia may involve impairment or incoordination of orpharyngeal functions, nasal regurgitation, poor control of the saliva, drooling, coughs, food stasis, and choking during feeding. $6,8,10,14,16-20$

The speech-language therapist should consider and recommend the most effective postural maneuvers to promote airway clearance and protection, and include advisement to caregivers in that which concerns volume, consistency, and pace of food administration. Neurological dysphagia comprises a wide variability of signs, symptoms, and prognoses. The selection of treatment strategies and techniques should be made according to scientific literature and guided by clinical findings on a patient-by-patient basis.

In general, CTM is effective in mild to moderate cases of dysphagia. Severe cases require a more aggressive approach, including the use of cuffs and enteral feeding during the rehabilitation process.

\section{Conclusion}

The effectiveness of CTM is related to the overall degree of dysphagia, the more severe the dysphagia, the less effective the maneuver. CTM should benefit dysphagic patients with pharyngeal delay time, reduced laryngeal elevation, and difficulties in swallowing liquids.

\section{References}

1 Ayuse T, Ayuse T, Ishitobi S, et al. Effect of reclining and chin-tuck position on the coordination between respiration and swallowing. J Oral Rehabil 2006;33(6):402-408

2 Cook IJ. Investigative techniques in the assessment of oral-pharyngeal dysphagia. Dig Dis 1998;16(3):125-133

3 Ding R, Larson CR, Logemann JA, Rademaker AW. Surface electromyographic and electroglottographic studies in normal subjects under two swallow conditions: normal and during the Mendelsohn maneuver. Dysphagia 2002;17(1):1-12 
4 Ertekin C, Keskin A, Kiylioglu N, et al. The effect of head and neck positions on oropharyngeal swallowing: a clinical and . electrophysiological study. Arch Phys Med Rehabil 2001;82(9): 1255-1260

5 Hori K, Tamine K, Barbezat C, et al. Influence of chin-down posture on tongue pressure during dry swallow and bolus swallows in healthy subjects. Dysphagia 2011;26(3):238-245

6 McCabe D, Ashford J, Wheeler-Hegland K, et al. Evidence-based systematic review: Oropharyngeal dysphagia behavioral treatments. Part IV-impact of dysphagia treatment on individuals' postcancer treatments. J Rehabil Res Dev 2009;46(2): 205-214

7 Ashford J, McCabe D, Wheeler-Hegland K, et al. Evidence-based systematic review: Oropharyngeal dysphagia behavioral treatments. Part III-impact of dysphagia treatments on populations with neurological disorders. J Rehabil Res Dev 2009;46(2): 195-204

8 Terré R, Mearin F. Effectiveness of chin-down posture to prevent tracheal aspiration in dysphagia secondary to acquired brain injury. A videofluoroscopy study. Neurogastroenterol Motil 2012;24(5):414-419, e206

9 Crary MA, Carnaby Mann GD, Groher ME, Helseth E. Functional benefits of dysphagia therapy using adjunctive sEMG biofeedback. Dysphagia 2004;19(3):160-164

10 Welch MV, Logemann JA, Rademaker AW, Kahrilas PJ. Changes in pharyngeal dimensions effected by chin tuck. Arch Phys Med Rehabil 1993;74(2):178-181
11 Logemann JA. Treatment of oral and pharyngeal dysphagia. Phys Med Rehabil Clin N Am 2008;19(4):803-816, ix

12 Macrae P, Anderson C, Humbert I. Mechanisms of airway protection during chin-down swallowing. J Speech Lang Hear Res 2014; 57(4):1251-1258

13 Solazzo A, Monaco L, Del Vecchio L, et al. Investigation of compensatory postures with videofluoromanometry in dysphagia patients. World J Gastroenterol 2012;18(23):2973-2978

14 Shanahan TK, Logemann JA, Rademaker AW, Pauloski BR, Kahrilas PJ. Chin-down posture effect on aspiration in dysphagic patients. Arch Phys Med Rehabil 1993;74(7):736-739

15 O'Neil KH, Purdy M, Falk J, Gallo L. The Dysphagia Outcome and Severity Scale. Dysphagia 1999;14(3):139-145

16 Chen MYM, Ott DJ, Peele VN, Gelfand DW. Oropharynx in patients with cerebrovascular disease: evaluation with videofluoroscopy. Radiology 1990;176(3):641-643

17 Horner J, Buoyer FG, Alberts MJ, Helms MJ. Dysphagia following brain-stem stroke. Clinical correlates and outcome. Arch Neurol 1991;48(11):1170-1173

18 Nagaya M, Kachi T, Yamada T, Sumi Y. Videofluorographic observations on swallowing in patients with dysphagia due to neurodegenerative diseases. Nagoya J Med Sci 2004;67(1-2):17-23

19 Leigh JH, Oh BM, Seo HG, et al. Influence of the chin-down and chin-tuck maneuver on the swallowing kinematics of healthy adults. Dysphagia 2015;30(1):89-98

20 Veis SL, Logemann JA. Swallowing disorders in persons with cerebrovascular accident. Arch Phys Med Rehabil 1985;66(6):372-375 